\title{
Design of $N$-hydroxysuccinimide Bifunctionalized Triblock Cross-Linker Having Hydrolysis Property for a Biodegradable and Injectable Hydrogel System
}

Shohei Ishikawa, ${ }^{\dagger}$ Daisuke Matsukuma, ${ }^{\ddagger}$ Kazutoshi Iijima,, \# Michihiro Iijima, " Shigehto

Osawa,,${ }^{*}$ and Hidenori Otsuka ${ }^{\dagger, *, *}$

${ }^{+}$Graduate School of Science, Tokyo University of Science, 1-3 Kagurazaka, Shinjuku-ku, Tokyo 162-8601, Japan.

${ }^{\ddagger}$ Department of Applied Chemistry, Faculty of Science, Tokyo University of Science, 1-3 Kagurazaka, Shinjuku-ku, Tokyo 162-8601, Japan.

${ }^{\S}$ Department of Ind ustrial Chemistry, Faculty of Engineering, Tokyo University of Science, 12-1 Ichigayafunagawara-machi, Shinjuku-ku, Tokyo 162-0826, Japan.

"Department of Materials Chemistry and Bioengineering, National Institute of Technology, Oyama College, 771 Nakakuki, Oyama, Tochigi 323-0806, Japan.

"Water Frontier Science \& Technology Research Center, Research Institute for Science and Technology, Tokyo University of Science, Shinjuku-ku, Tokyo 162-8601 Japan.

\#Present address: Faculty of Engineering, Yokohama National University, 79-5 Tokiwadai, Hodogaya-ku, Yokohama 240-8501, Japan.

*Correspondence: osawa-s@rs.tus.ac.jp and h.otsuka@rs.kagu.tus.ac.jp. 
Supporting Information.

\section{SI.1 Synthesis of control linkers.}

\section{Method}

\section{Synthesis of OH-PLA-b-PEG-b-PLA-OH}

End-hydrophobic PLA- $b$-PEG- $b$-PLA was synthesized by ring-opening polymerization of DL-lactide using OH-PEG-OH as an initiator. PEG (2.0 g, $1 \mathrm{mmol})$, DL-lactide $1440 \mathrm{mg}, 10$ mmol, 10 eq. vs. $\mathrm{PEG}$ ), and $\mathrm{Sn}(\mathrm{Oct})_{2}(405 \mathrm{mg}, 0.1 \mathrm{mmol}, 0.1$ eq. vs. $\mathrm{PEG}$ ) were added into a two-necked flask under nitrogen atmosphere. The reaction was allowed to proceed under refluxing for $48 \mathrm{~h}$ at $120^{\circ} \mathrm{C}$. The mixture was concentrated and reprecipitated three times with chloro form in cold diethyl ether, and then freeze-dried from benzene. Molecular weight of PLA was characterized by ${ }^{1} \mathrm{H}-\mathrm{NMR}$ measurement.

\section{Synthesis of bifunctional PLA- $b$-PEG- $b$-PLA and PEG with succinimidyl carbonate}

PLA- $b$-PEG- $b$-PLA $(2.0 \mathrm{~g}, 0.7350 \mathrm{mmol})$ was dissolved in $50 \mathrm{~mL}$ of dichloromethane and stirred for $10 \mathrm{~min}$ under nitrogen atmosphere. Separately, DSC (940 mg, $3.7 \mathrm{mmol})$ was dissolved in $20 \mathrm{~mL}$ dry acetonitrile at $50{ }^{\circ} \mathrm{C}$ for $30 \mathrm{~min}$. Then, pyridine $(200 \mu \mathrm{L}, 2.5 \mathrm{mmol})$ was added to reaction mixture. After $24 \mathrm{~h}$ stirring at room temperature, the mixture was filtered and dried up in vacuum. The sample was dissolved in $10 \mathrm{~mL}$ of chloroform and poured into $300 \mathrm{~mL}$ of cold diisopropyl ether to precipitate the product. After repeating the precipitation twice, the sample was dissoved in $5 \mathrm{~mL}$ of benzene and freeze-dried to collect as a white powder (NHS-PLA- $b$-PEG- $b$-PLA-NHS). NHS substitution was confirmed by ${ }^{1} \mathrm{H}-\mathrm{NMR}$.

OH-PEG-OH was converted to NHS-PEG-NHS in the same manner as mentionded the above. PEG (2.0 g, $0.4 \mathrm{mmol}$ ) was dissolved in $50 \mathrm{~mL}$ of dichloromethane and stirring for $10 \mathrm{~min}$ under nitrogen atmosphere. Separately, DSC (510 mg, $2.0 \mathrm{mmol})$ was dissolved in $20 \mathrm{~mL}$ dry acetonitrile at $50^{\circ} \mathrm{C}$ for $30 \mathrm{~min}$. After mixing these solutions, pyridine $(200 \mu \mathrm{L}, 2.5 \mathrm{mmol})$ was added to reaction mixture. After $24 \mathrm{~h}$ stirring at room temperature, the mixture was filtered and dried up in vacuum. The sample was dissolved in $10 \mathrm{~mL}$ of chloroform and poured into $300 \mathrm{~mL}$ of cold diisopropyl ether to precipitate the product. After repeating the precipitation twice, the sample was dissoved in $5 \mathrm{~mL}$ of benzene and freeze-dried to collect as a white powder (NHS-PEG-NHS). NHS substitution was confirmed by ${ }^{1} \mathrm{H}-\mathrm{NMR}$. 
Results
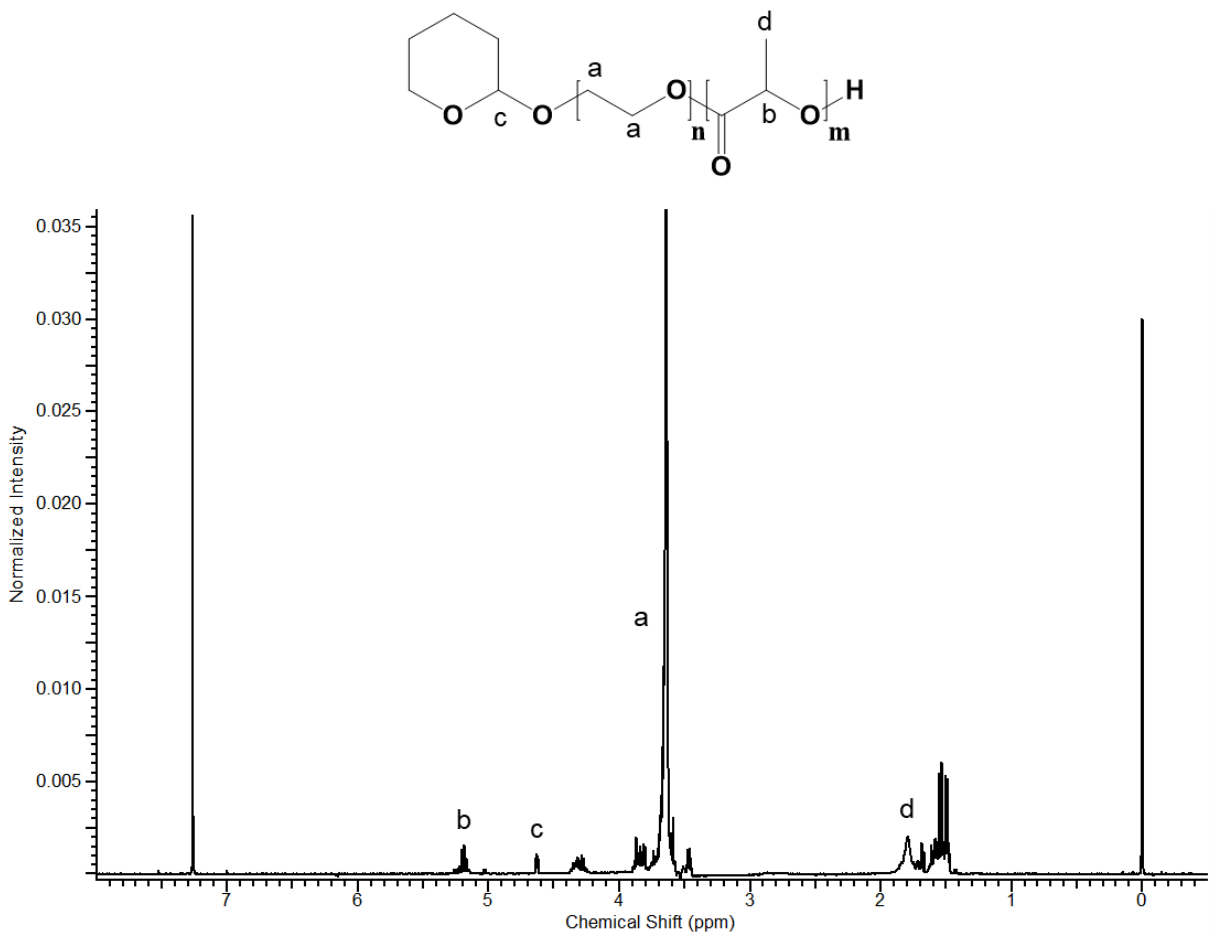

Figure S1. ${ }^{1} \mathrm{H}-\mathrm{NMR}$ spectrum of THP-PEG- $b$-PLA-OH.
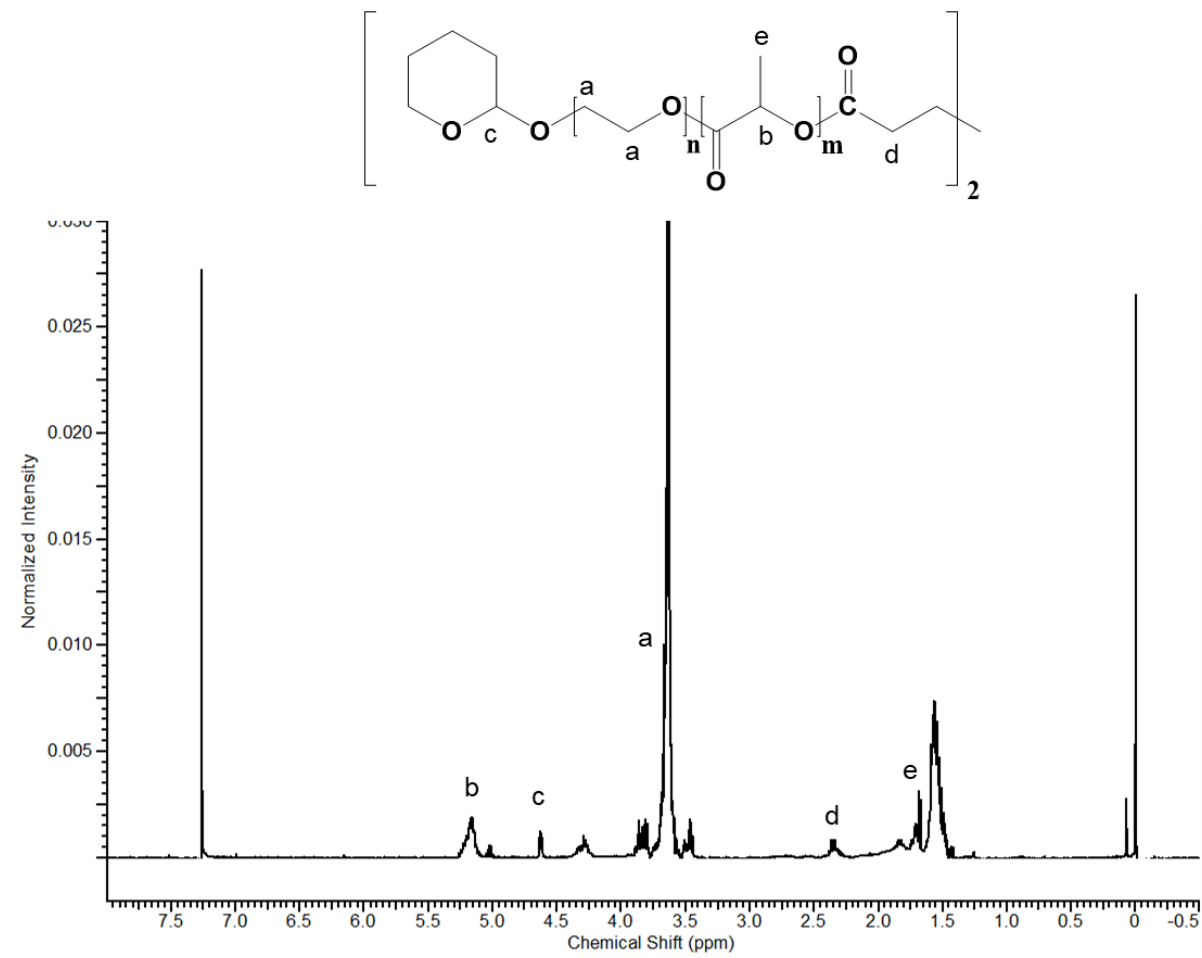

Figure S2. ${ }^{1} \mathrm{H}-\mathrm{NMR}$ spectrum of THP-PEG- $b$-PLA- $b$-PEG-THP. 

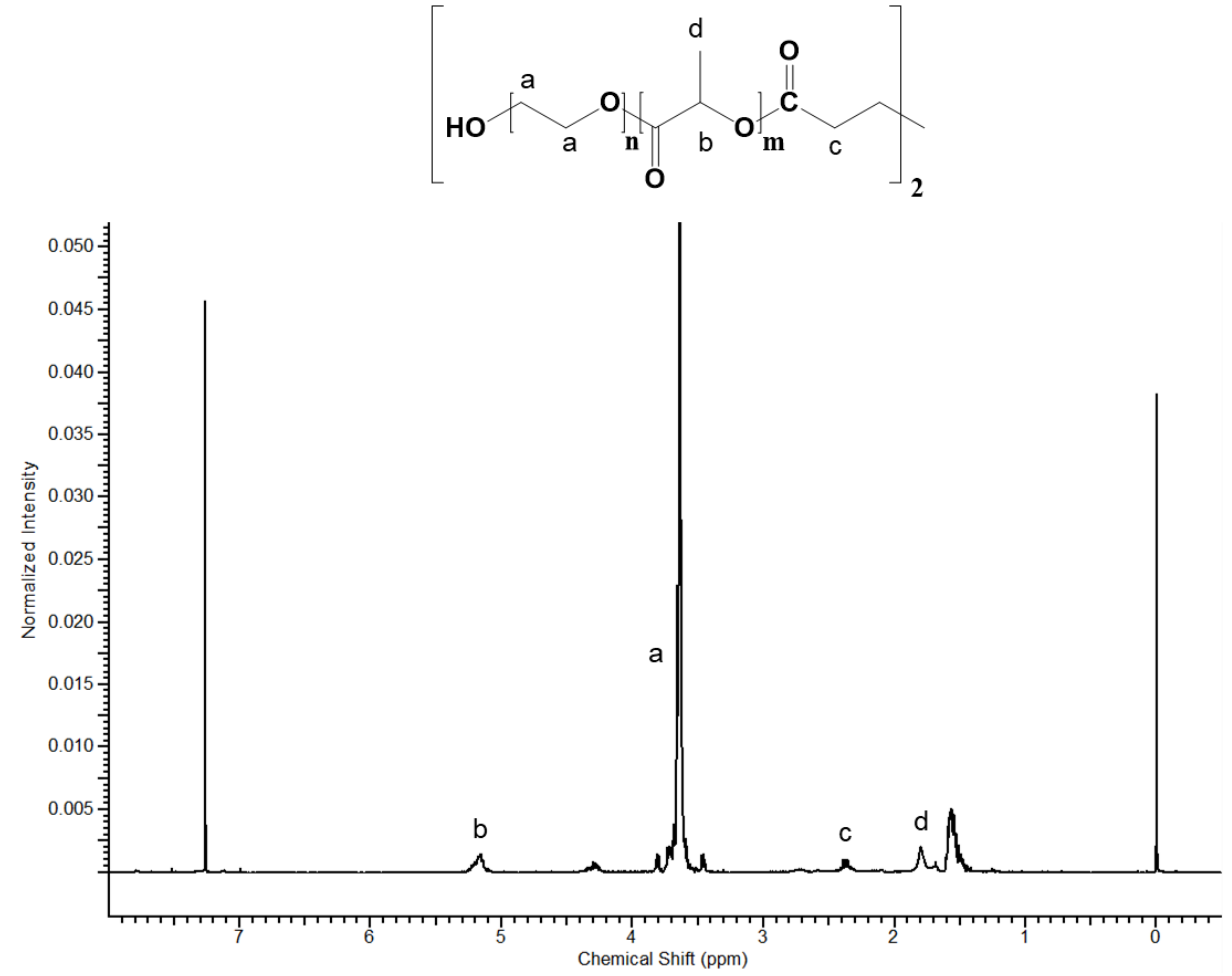

Figure S3. ${ }^{1} \mathrm{H}-\mathrm{NMR}$ spectrum of $\mathrm{OH}-\mathrm{PEG}-b$-PLA- $b$-PEG-OH.

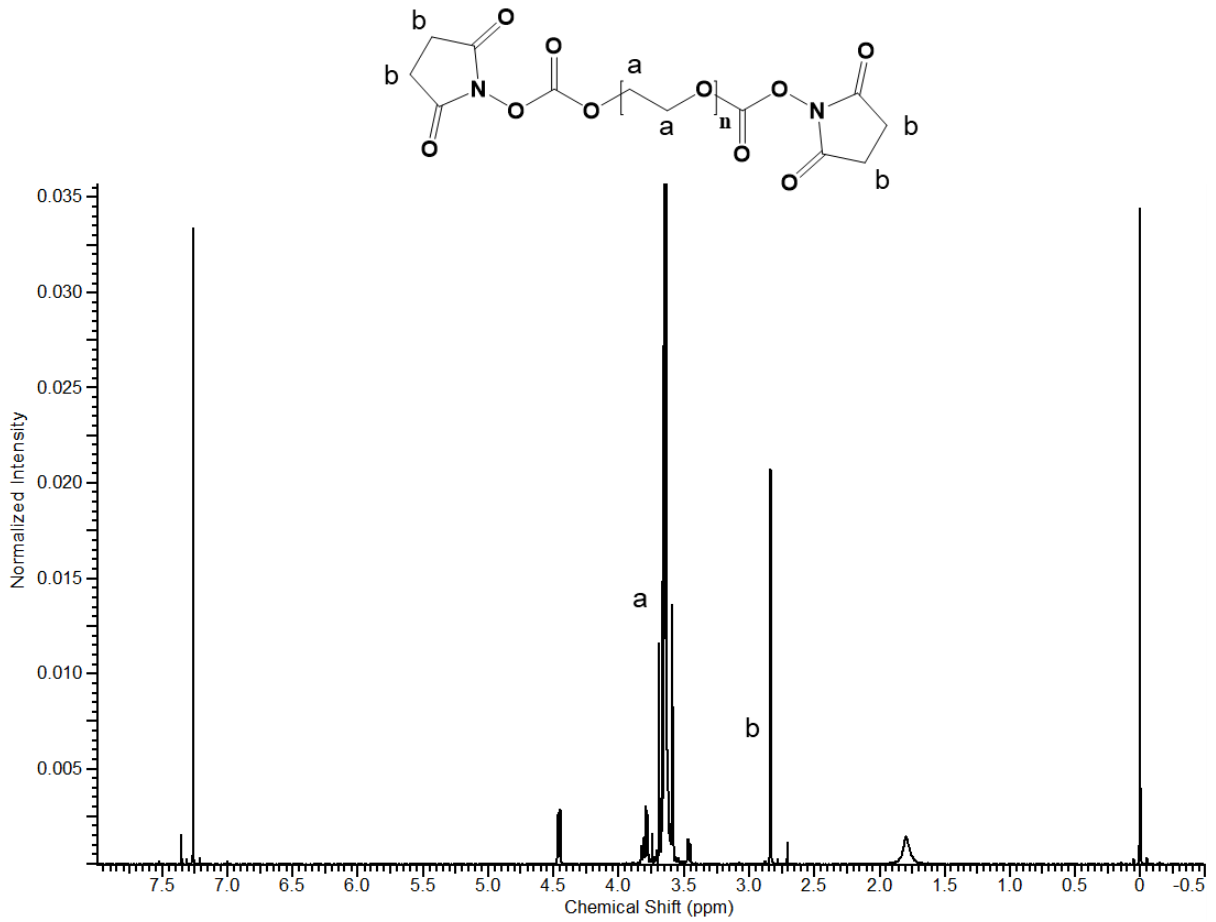

Figure S4. ${ }^{1} \mathrm{H}-\mathrm{NMR}$ spectrum of NHS-PEG-NHS. 


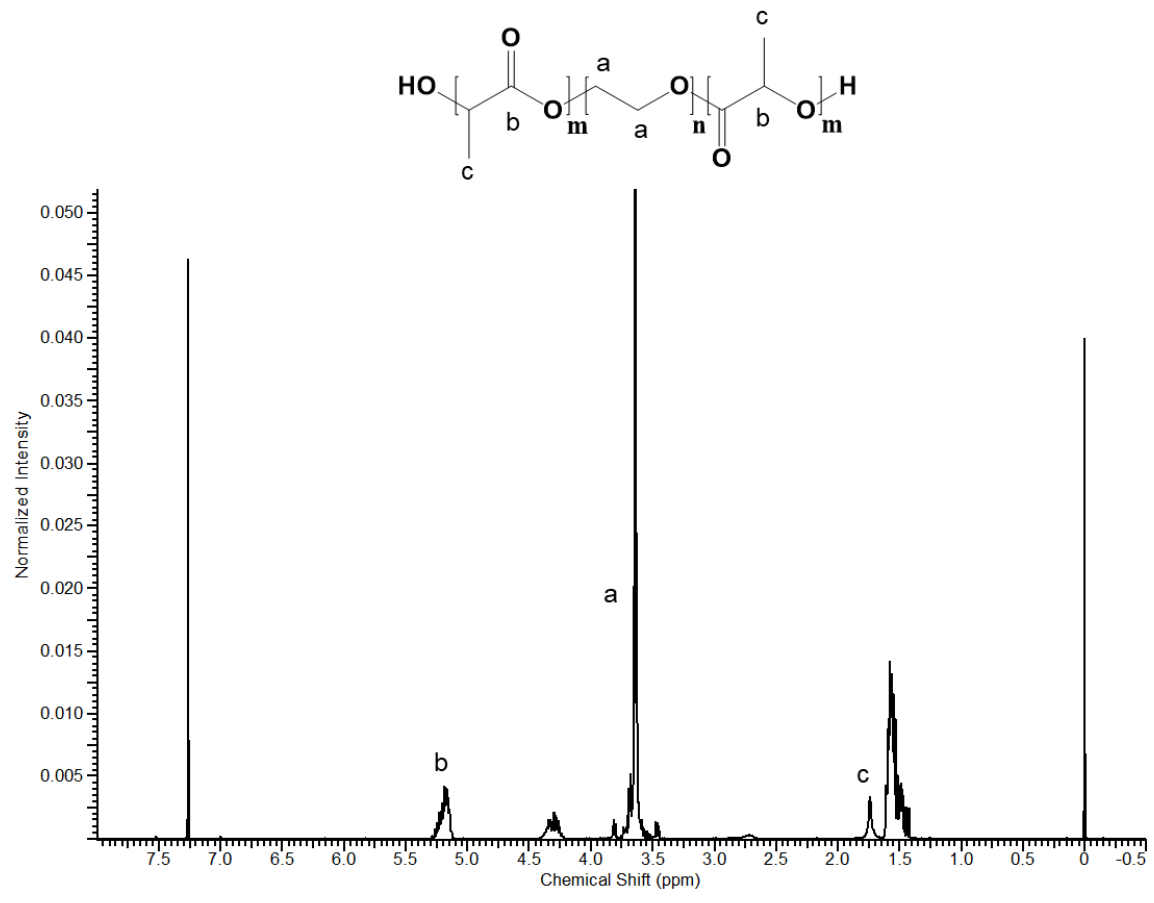

Figure S5. ${ }^{1} \mathrm{H}-\mathrm{NMR}$ spectrum of OH-PLA- $b$-PEG- $b$-PLA-OH.

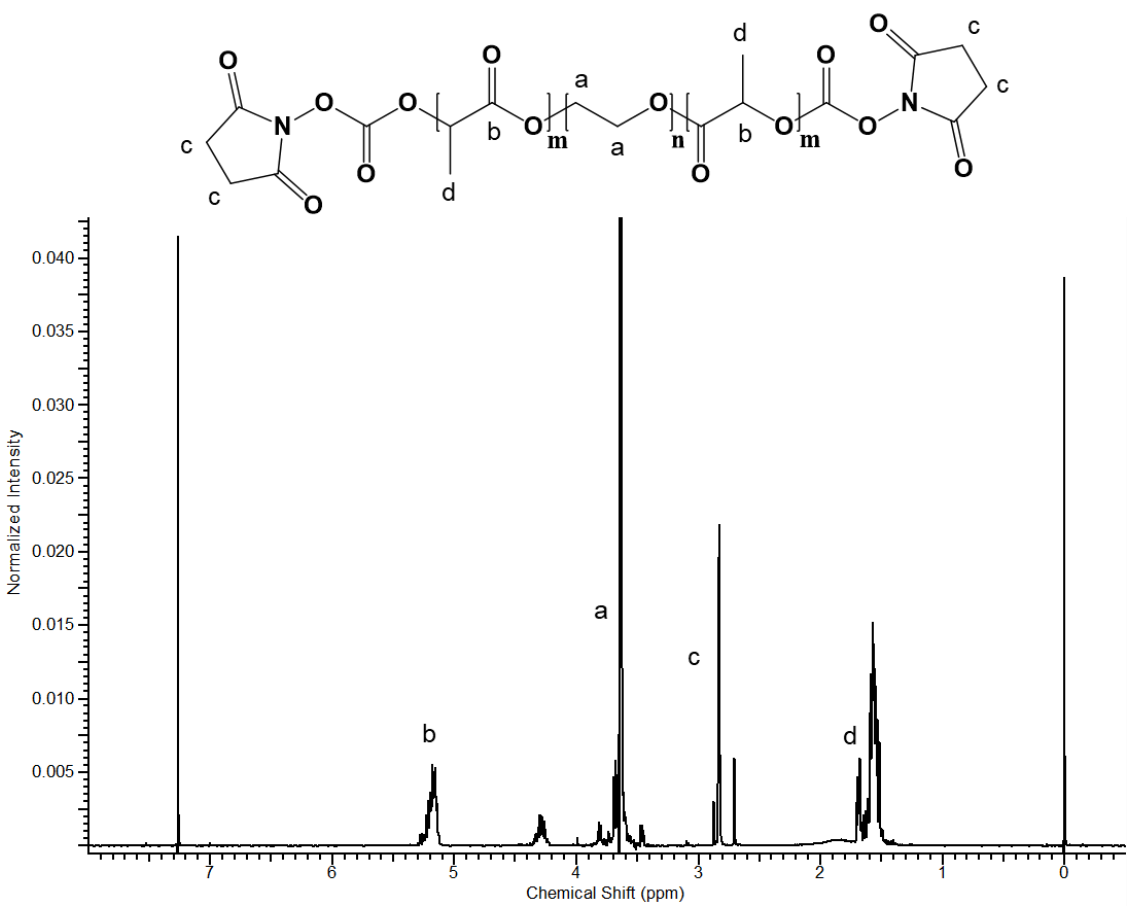

Figure S6. ${ }^{1} \mathrm{H}-\mathrm{NMR}$ spectrum of NHS-PLA- $b$-PEG- $b$-PLA-NHS. 


\section{SI.2 Evaluation of critical micelle concentration of PEG- $b$-PLA- $b$-PEG.}

\section{Method}

Thirty $\mu \mathrm{L}$ of a $1 \mathrm{mM}$ 1-pyrenecarboxaldehyde (PCA, Wako, Japan) dissolved in ethanol was prepared and dried up in vacuum to produce microcrystal of PCA. Then, $3 \mathrm{~mL}$ of OH-PEG- $b$-PLA- $b$-PEG-OH solutions dissolved in $150 \mathrm{mM}$ PBS $(0.001,0.01,0.1,1$, and 5 $\mathrm{wt} \%$ ) were added, and the solutions were shaked for $24 \mathrm{~h}$. The position of the fluorecence peak maximum (ex. $370 \mathrm{~nm}$ ) was tracked using a spectrofluorometer (FP-6500, JASCO, Japan) ${ }^{\mathrm{S} 1}$.

Result

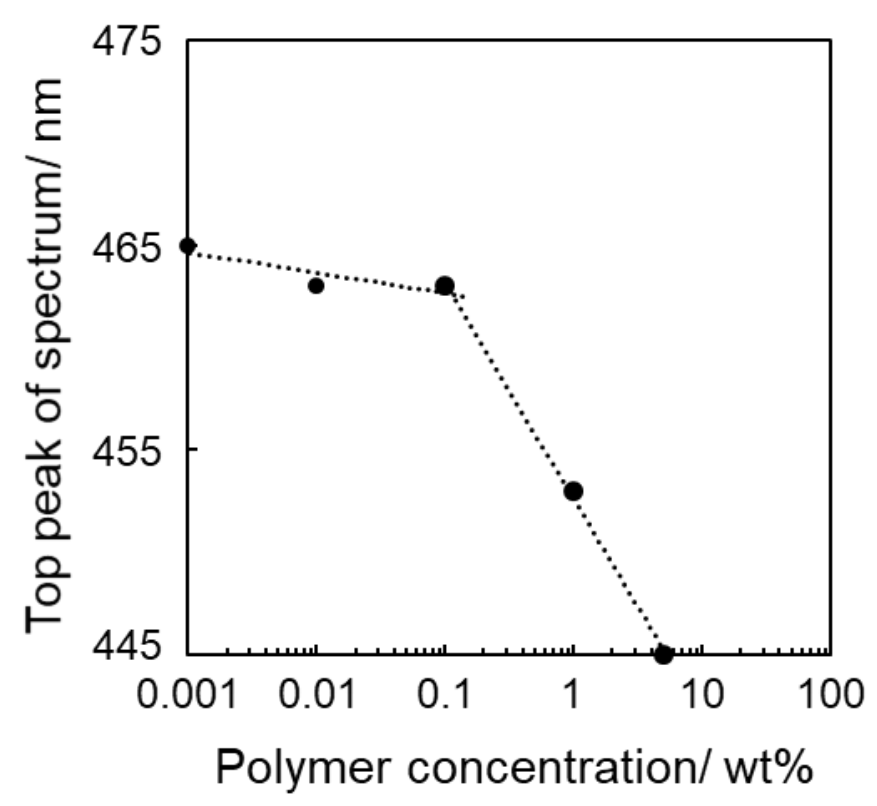

Figure S7. The position of the fluorescence peak maximum of PCA solutions with various PEG-PLA-PEG concentrations. Critical micelle concentration (CMC) of PEG- $b$-PLA- $b$-PEG were determined to be $0.1 \mathrm{wt} \%$. 


\section{with linkers.}

\section{Method}

2,4,6-trinitrobenzenesulfonic acid sodium salt dehydrate (TNBS) (Wako, Japan) was used to evaluate consumption of the primary amine in $\mathrm{CH}$ before and after mixing with linkers as previously described ${ }^{\mathrm{S} 2}$. A hundred $\mu \mathrm{L}$ of $\mathrm{CH} / \mathrm{PEG}$ and $\mathrm{CH} / \mathrm{PEG}-\mathrm{PLA}-\mathrm{PEG}$ hydrogels were prepared by mixing $50 \mu \mathrm{L}$ of $4 \mathrm{wt} \% \mathrm{CH}$ solution, $25 \mu \mathrm{L}$ of $4 \mathrm{wt} \%$ linker solution and $25 \mu \mathrm{L}$ of PBS on 96-well culture plate (Corning, NY, USA) in the same manner as described in the main text. Then, $100 \mu \mathrm{L}$ of $4 \mathrm{wt} \%$ sodium hydrogen carbonate (Wako, Japan) and $100 \mu \mathrm{Lof} 0.1 \mathrm{wt} \%$ TNBS aqueous solutions were added to these hydrogels. After incubation at $37{ }^{\circ} \mathrm{C}$ for 60 minutes, absorbance at $350 \mathrm{~nm}$ was measured using a microplate reader (Varioskan Flash, Thermo Fisher Scientific, USA). A hundred $\mu \mathrm{L}$ of $2 \mathrm{wt} \% \mathrm{CH}$ solution was also treated on 96 wells plate in the same manner as the above description to prepare a control without mixing cross-linkers.

Results

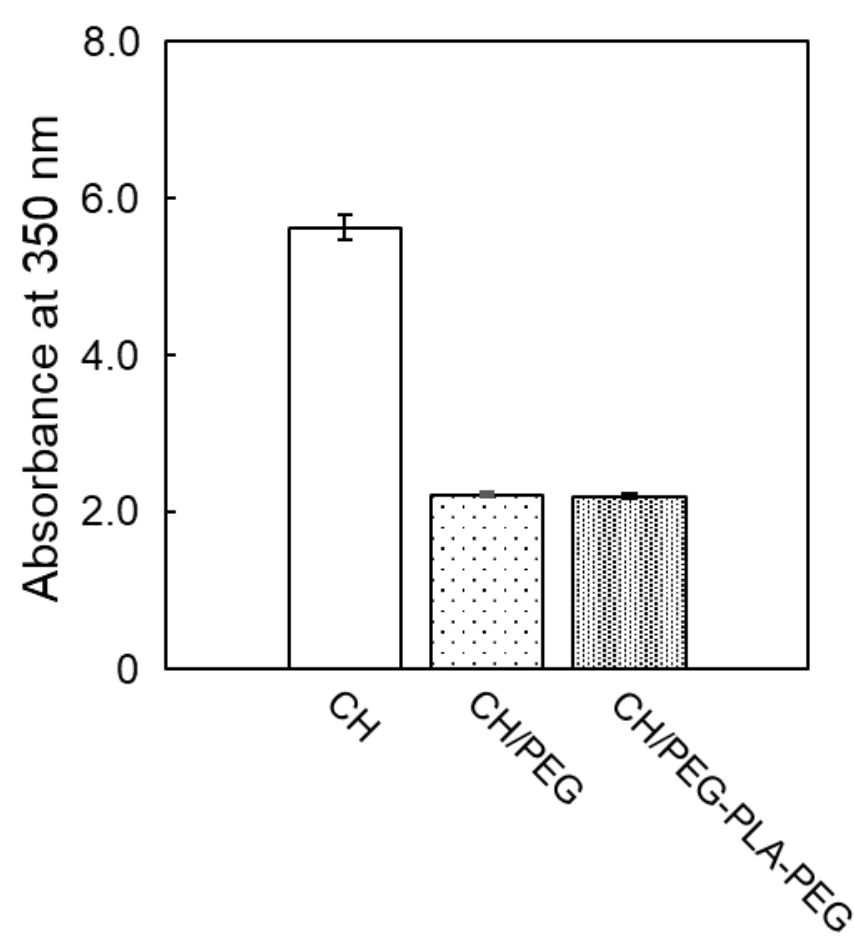

Figure S8. Absorbance at $350 \mathrm{~nm}$ of $\mathrm{CH}$ treated by TNBS with or without cross-linkers.

Data are shown as the mean \pm the standard error of the mean (S. E., $n=2)$.

\section{Reference}

S1. Ananthapadmanabhan, K. P.; Goddard, E. D.; Turro N. J.; Kuo P. L. Fluorescence probes for critical micelle concentration. Langmuir, 1985, 1, 352-355. 
S2. Müller, A.; Langklotz, S.; Lupilova, N.; Kuhlmann, K.; Bandow, J. E.; Leichert, L. I. Activation of RidA chaperone function by N-chlorination. Nat. Commun. 2014, 5, 5804. DOI: $10.1038 /$ ncomms6804. 